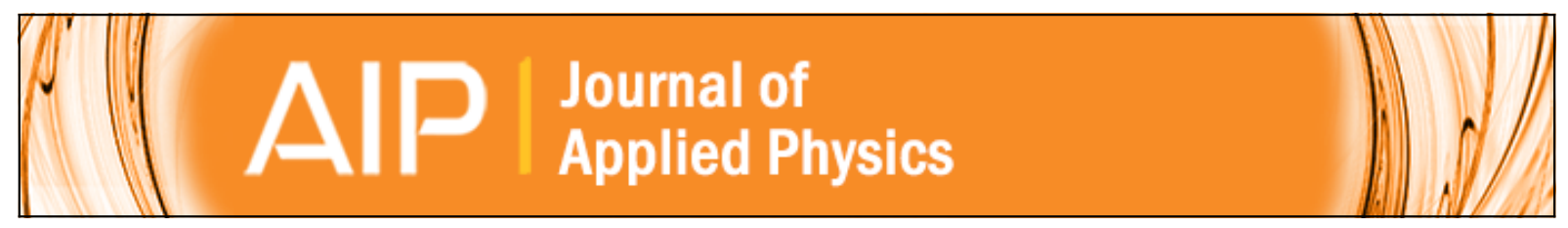

\title{
Orbiting atoms and $\mathrm{C} \mathbf{6 0}$ fullerenes inside carbon nanotori
}

Tamsyn A. Hilder and James M. Hill

Citation: Journal of Applied Physics 101, 064319 (2007); doi: 10.1063/1.2511490

View online: http://dx.doi.org/10.1063/1.2511490

View Table of Contents: http://scitation.aip.org/content/aip/journal/jap/101/6?ver=pdfcov

Published by the AIP Publishing

\section{Articles you may be interested in}

Oscillatory characteristics of carbon nanotubes inside carbon nanotube bundles

J. Appl. Phys. 112, 124310 (2012); 10.1063/1.4770327

Diffusive growth of fullerenes and carbon nanotubes

J. Chem. Phys. 131, 244703 (2009); 10.1063/1.3277673

Oscillatory behavior of gigahertz oscillators based on multiwalled carbon nanotubes

J. Appl. Phys. 98, 014301 (2005); 10.1063/1.1942648

Inserting Fullerene Dimers into Carbon Nanotubes: Pushing the Boundaries of Molecular Self-assembly AIP Conf. Proc. 723, 255 (2004); 10.1063/1.1812085

Vibrational behaviors of multiwalled-carbon-nanotube-based nanomechanical resonators

Appl. Phys. Lett. 84, 121 (2004); 10.1063/1.1638623

\section{AlP $\mid$ APL Photonics}

APL Photonics is pleased to announce Benjamin Eggleton as its Editor-in-Chief

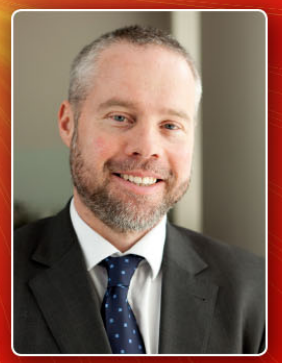




\title{
Orbiting atoms and $\mathrm{C}_{60}$ fullerenes inside carbon nanotori
}

\author{
Tamsyn A. Hilder and James M. Hill \\ Nanomechanics Group, School of Mathematics and Applied Statistics, University of Wollongong, \\ Wollongong NSW 2522, Australia
}

(Received 10 May 2006; accepted 29 December 2006; published online 29 March 2007)

\begin{abstract}
The discovery of carbon nanostructures, such as carbon nanotubes and $\mathrm{C}_{60}$ fullerenes, has generated considerable interest for potential nanoelectronic applications. One such device is the high frequency nanoscale gigahertz oscillator. Several studies investigating these oscillators demonstrate that sliding an inner-shell inside an outer-shell of a multiwalled carbon nanotube generates oscillatory frequencies in the gigahertz range. Research has shown that the oscillation is sensitive to the diameter and the helicity of the tube and that the inner tube length can be used to tune the frequency, such that the smaller the inner tube length the higher the frequency of oscillation, suggesting that a $\mathrm{C}_{60}$ fullerene might provide the ultimate core. Recently, researchers have observed single continuous toroidal nanotubes with no beginning or end, effectively a single-walled carbon nanotube closed around onto itself so that the two open ends fuse together, stabilized by van der Waals forces alone, to form a perfect "nanotorus." The question arises as to whether it is possible to create a $\mathrm{C}_{60}$ - nanotorus oscillator or orbiter, comprising a $\mathrm{C}_{60}$ fullerene orbiting around the inside of a nanotorus. The $\mathrm{C}_{60^{-}}$nanotorus orbiter has yet to be constructed and the aim here is to assess its feasibility by examining the dominant mechanics of this potential nanoscale device. As in previous studies, the Lennard-Jones potential is used to calculate the interatomic forces acting on the fullerene due to the nonbonded interactions. Furthermore, other relevant forces are examined. Initially, we investigate the dynamics of an orbiting single atom followed by the corresponding analysis for an orbiting $\mathrm{C}_{60}$ fullerene. The equilibrium position depends on the radius of the nanotorus tube for both the atom and the $\mathrm{C}_{60}$ fullerene. Gravity is shown to be negligible, while the centrifugal forces are shown to move the orbiting body further from the center of the nanotorus. The theory also predicts that by changing the orbital position, the resulting frequencies, which are in the gigahertz range, may vary to as much as four times those obtained for the $\mathrm{C}_{60}$-nanotube oscillator.

(C) 2007 American Institute of Physics. [DOI: 10.1063/1.2511490]
\end{abstract}

\section{INTRODUCTION}

The discovery by Iijima ${ }^{1}$ in 1991 illustrating that carbon nanotubes could be synthesized without the need for a catalyst, paved the way for numerous proposals for nanoscale devices. Carbon nanotubes may be thought of as one or many graphene sheets rolled up into a seamless hollow cylinder, forming single-wall (SWNT) or multiwall (MWNT) carbon nanotubes, respectively. They have many fascinating and unique mechanical and electronic properties, including but not limited to, their high strength and flexibility, low density, completely reversible deformation and their ability to be metallic or semiconducting depending on their geometric structure. Both SWNT and MWNT hold promise for many new nanoelectronic applications. The potential use of carbon nanotubes as high frequency nanoscale oscillators is one demonstration of their outstanding properties. Micromechanical oscillators are unable to reach frequencies in the gigahertz range, however, nanoscale oscillators are able to achieve these high frequencies and have, therefore, been termed gigahertz oscillators. Potential applications of these gigahertz oscillators include ultrafast optical filters for fiber optic systems, nano-antennae sensitive to high frequency electromagnetic signals and increasing the computer chip's speed in processing electronic signals.

Considerable research has been undertaken to further un- derstand carbon nanotube's unique properties. In an experiment investigating the strength and breaking mechanism of the MWNT under tensile load, Yu et al. ${ }^{2}$ observe low shear strength between layers. Cumings and Zettl ${ }^{3}$ subsequently investigate this result by controlled and reversible extrusion of the inner-shell, and demonstrate that the inner-shell resistance force against sliding of the core is negligibly small, realizing ultralow friction. Following this, Zheng and Jiang ${ }^{4}$ propose the idea of a nano-oscillator, in which the inner-shell oscillates inside the outer-shell of a MWNT. Mathematical analysis and molecular-dynamics simulations ${ }^{4,5}$ show that oscillatory frequencies in the gigahertz range are generated. Legoas et al..$^{5}$ observe frequencies as high as $38 \mathrm{GHz}$. Furthermore, Zheng and Jiang ${ }^{4}$ observe that decreasing the length of the inner tube can further increase the oscillation frequency. The inner tube length can, therefore, be used to tune the oscillation frequency and a $\mathrm{C}_{60}$ fullerene might provide the ultimate core in terms of realizing the highest possible frequency of oscillation.

These remarkable results combined with the discovery of peapods, ${ }^{6}$ which are $\mathrm{C}_{60}$ fullerenes (buckyballs) encapsulated in carbon nanotubes, has motivated the interest in the $\mathrm{C}_{60}$-nanotube oscillator, ${ }^{7,8}$ comprising a buckyball oscillating inside a carbon nanotube. Molecular-dynamics simulations indicate that frequencies as high as $74 \mathrm{GHz}$ may be obtained, ${ }^{7}$ and that the oscillation amplitude is almost con- 
stant with no sign of decay. ${ }^{9}$ Such studies find that the oscillation is sensitive to both the diameter and the helicity of the tube (the orientation of the hexagonal units with respect to the tube axis). Specific combinations of nanotube diameter and buckyball diameter are found to minimize frictional effects, ${ }^{7}$ and the minimum potential energy of the system occurs when the distance between the tube and the buckyball is close to the interlayer distance of graphite. ${ }^{8}$ There also exists a minimum radius $(6.27 \AA)$ of the nanotube that may be filled with $\mathrm{C}_{60}$ fullerenes. ${ }^{8}$ In previous studies ${ }^{6,9}$ of the $\mathrm{C}_{60}$-nanotube oscillator, it is shown that under certain circumstances the $\mathrm{C}_{60}$ fullerene may be sucked into the nanotube due to the highly attractive interatomic van der Waals forces. However, not all carbon nanotubes demonstrate this suction behavior. Cox, Thamwattana, and $\mathrm{Hill}^{10}$ formulate an acceptance condition, which prescribes whether or not the buckyball will be sucked into the nanotube by van der Waals forces alone. A fundamental practical issue is to determine an experimental procedure to set in motion these ultrahigh frequency oscillators in a controlled manner.

Recently, toroidal carbon nanotubes, termed fullerene "crop circles," have been observed in experiments. ${ }^{11}$ Circular formations of SWNT ropes are regularly observed while examining laser-grown SWNT. Continuous toroidal nanotubes, with no beginning or end, with a tube diameter of approximately $10-12 \AA$ and a ring diameter of approximately 3000 $5000 \AA$ are observed. After the ends of the tube touch, they align themselves due to the van der Waals interaction energy, knitting together seamlessly to form a perfect torus. Once formed, the rings are quite stable both chemically and physically. ${ }^{12}$ Martel, Shea, and Avouris ${ }^{13}$ form rings using straight SWNT whose ends fuse onto themselves so that the ring circumference is equal to the initial tube length. These ring ends stabilize by van der Waals forces alone. ${ }^{14}$ MWNTs are found to be less likely to form rings as they have a much larger diameter and a much higher flexural rigidity. The critical ring radius necessary to form thermodynamically stable rings is found to be $300 \AA$ for single-walled tubes $14 \AA$ in diameter. ${ }^{14}$ According to Martel, Shea, and Avouris ${ }^{14}$ much lower values of ring radius are energetically allowable than are actually observed, indicating that ring formation may be kinetically controlled. Using molecular-dynamics simulations Huhtala et al. ${ }^{15}$ and $\mathrm{Han}^{16}$ investigate the stability of these toroidal carbon nanotubes and also find much smaller ring diameters are possible than those observed experimentally. For example, Huhtala et al. ${ }^{15}$ find that a ring diameter of $220 \AA$ must have a tube diameter below $13 \AA$ for the nanotorus to remain stable. Similarly, $\operatorname{Han}^{16}$ finds ring diameters must be greater than 100,200 , and $400 \AA$ for a nanotorus $(5,5),(8,8)$, and $(10,10)$, respectively, to remain energetically stable. Effectively, the toroidal nanotube structure can be thought of as a SWNT closed around onto itself into a perfect torus. In this paper the toroidal SWNT are referred to as nanotori.

The question arises as to whether it might be possible to close a nanotube into a nanotorus that already contains an oscillating $\mathrm{C}_{60}$ fullerene, or alternatively to inject a $\mathrm{C}_{60}$ fullerene into a torus just prior to closure and subsequently initiate the orbiting motion. For example, could this motion be effected by application of an electric field, or by a variable magnetic field or by chemical doping? Such techniques pose many practical challenges that will need to be overcome before an actual $\mathrm{C}_{60}$-nanotorus oscillator or orbiter can be realized.

It is probable that the $\mathrm{C}_{60}$-nanotorus orbiter will display the ultralow friction demonstrated by Cumings and Zettl, ${ }^{3}$ and if so, the buckyball might orbit almost indefinitely inside the nanotorus. A sealed structure is ideal in terms of working devices, therefore, the $\mathrm{C}_{60}$-nanotorus orbiter may well be the ultimate oscillator. $\mathrm{A}_{60}$-nanotorus orbiter has yet to be constructed and the aim here is to assess its feasibility from a consideration of the basic mechanics of such a system. Nanoscale oscillatory systems have been predominantly studied using molecular-dynamics simulations. In this paper we use elementary mechanical principles and classical applied mathematical modeling techniques, following those formulated by Cox, Thamwattana, and Hill. ${ }^{20}$ Despite the speculative nature of these potential nanoscale devices, such a study must necessarily precede any practical implementation.

Following Cumings and Zettl ${ }^{3}$ and as an initial attempt to model this system we ignore any frictional effects. Although we consider the effect of gravity, we show that for a horizontally inclined nanotorus the effects of gravity are considerably less than those arising from the Lennard-Jones potential and the centrifugal effect, and accordingly gravity may be neglected. Both the offset atom and the buckyball minimum energy (equilibrium) positions are found to depend on the nanotorus tube radius. This equilibrium position moves closer to the tube wall as the nanotorus tube radius increases. The centrifugal effect is shown to shift the equilibrium position of both the atom and the buckyball away from the center of the nanotorus ring and closer to the tube wall. The frequencies of the orbiting motion are found to be in the gigahertz range, and increase as the position of the orbiting body moves away from the center of rotation. These predicted frequencies may vary as much as four times those obtained by the $\mathrm{C}_{60}$-nanotorus oscillator.

In this paper we investigate the mechanics of the motion of a single offset atom and following this we examine the motion of a $\mathrm{C}_{60}$ fullerene, both of which are assumed to be orbiting inside a single-walled carbon nanotorus. The following section outlines the Lennard-Jones potential, which is widely used to determine the interatomic forces in the modeling of nonbonded molecular interactions. Subsequently, a summary of the forces acting on the rotating body, such as gravity and the centrifugal force is given. The analysis for the minimum energy location of an offset atom, followed by a similar analysis for a $\mathrm{C}_{60}$ fullerene inside a nanotorus are given in Secs. III and IV, respectively. Numerical results for the angular velocity of the orbiting $\mathrm{C}_{60}$ molecule inside the nanotorus are outlined in Sec. V and the major implications of the model are summarized in Sec. VI.

\section{MECHANICAL CONSIDERATIONS}

In this section a summary of the Lennard-Jones potential and numerical values of the relevant model parameters are 
TABLE I. Constants used in the model

\begin{tabular}{ll}
\hline \hline${\text { Radius of } \mathrm{C}_{60}}$ & $a=3.55 \AA$ \\
Radius $^{\mathrm{a}}$ of $(6,6)$ & $b=4.071 \AA$ \\
Radius $^{\mathrm{a}}$ of $(10,10)$ & $b=6.78 \AA$ \\
Radius $^{\mathrm{a}}$ of $(16,16)$ & $b=10.856 \AA$ \\
Carbon-carbon bond length & $\sigma=1.42 \AA$ \\
Mean surface density of graphene & $\eta_{g}=4 \sqrt{3} /\left(9 \sigma^{2}\right)$ atoms $/ \AA^{2}$ \\
Mean surface density of buckyball & $\eta_{b}=60 /\left(4 \pi a^{2}\right)$ atoms $/ \AA^{2}$ \\
Mass of $\mathrm{C}_{60}$ & $M=16 \pi \sqrt{3} \mathrm{a}^{2} \mathrm{~m}_{0}^{2} /\left(9 \sigma^{2}\right)=60 m_{0}$ \\
Mass of single carbon atom & $m_{0}=12 \times 1.661 \times 10^{-27} \mathrm{~kg}$ \\
Attractive constant & $A=17.4 \mathrm{eV} \times \AA^{6}$ \\
Repulsive constant & $B=29 \times 10^{3} \mathrm{eV} \times \AA^{12}$ \\
\hline
\end{tabular}

${ }^{\mathrm{a}}$ Reference 17.

given. The other forces acting on the orbiting body are outlined, followed by an explanation of the initial orbital velocity used in the numerical calculations.

\section{A. Lennard-Jones potential function}

The nonbonded interaction energy is obtained by a summation of the interaction energy between each atom pair

$$
V_{1}=\sum_{i} \sum_{j} v\left(\rho_{i j}\right)
$$

where $v\left(\rho_{i j}\right)$ is the potential function for atoms $i$ and $j$ at a distance $\rho_{i j}$ apart. Following conventional practice, the continuum approximation assumes that the atoms are uniformly distributed over the surface of the molecule and the double summation in Eq. (2.1) is replaced by a double integral over the surface of each molecule, thus

$$
V_{1}=\eta_{b} \eta_{t} \iint v(\rho) d \Sigma_{b} d \Sigma_{t},
$$

where $\eta_{b}$ and $\eta_{t}$ represent the mean surface density of the carbon atoms on the buckyball and nanotorus, respectively, and $\rho$ represents the distance between the two typical surface elements $d \Sigma_{b}$ and $d \Sigma_{t}$ located on the two interacting molecules, in this case the $\mathrm{C}_{60}$ fullerene and the torus, respectively. Table I gives the numerical values for the various constants used in the model. Note that the mean surface density of the nanotorus is taken to be equal to that of graphene.

There are two major functional forms used in empirical models: The inverse power model and the Morse function model. $9,17,18$ The inverse power model, the so-called Lennard-Jones potential, is adopted in this investigation. The Lennard-Jones potential for two atoms a distance $\rho$ apart is

$$
\nu(\rho)=-A \rho^{-6}+B \rho^{-12},
$$

where $A$ and $B$ are known as the attractive and repulsive constants, respectively, and are given in Table I. The equilibrium distance, $\rho_{0}$ between an atom pair is given by $\rho_{0}$ $=(2 B / A)^{1 / 6}$.

The Lennard-Jones potential has been used for a number of molecular configurations, and examples include two identical parallel carbon nanotubes, ${ }^{6}$ between two $\mathrm{C}_{60}$ fullerenes, ${ }^{19}$ and between a carbon nanotube and $\mathrm{C}_{60}$ (both inside and outside the tube). ${ }^{6,9}$ Numerical values of the Lennard-Jones constants for atoms in graphene-graphene,
TABLE II. Lennard-Jones constants for graphitic systems. ${ }^{\mathrm{a}}$

\begin{tabular}{lccc}
\hline \hline & $A\left(\mathrm{eV} \times \AA^{6}\right)$ & $B\left(\mathrm{eV} \times \AA^{12}\right)$ & $\rho_{0}(\AA)$ \\
\hline Graphene-graphene & 15.2 & $24.1 \times 10^{3}$ & 3.83 \\
$\mathrm{C}_{60}-\mathrm{C}_{60}$ & 20.0 & $34.8 \times 10^{3}$ & 3.89 \\
$\mathrm{C}_{60}$-graphene & 17.4 & $29.0 \times 10^{3}$ & 3.86 \\
\hline \hline
\end{tabular}

${ }^{\mathrm{a}}$ Reference 6 .

$\mathrm{C}_{60}-\mathrm{C}_{60}$ and $\mathrm{C}_{60}$-graphene are shown in Table II. Note that in this investigation we use Lennard-Jones constants for the $\mathrm{C}_{60}$-graphene case.

\section{B. Force balance for orbiting motion}

There are three forces acting on the orbiting atom or fullerene, the van der Waals force modeled by the LennardJones potential, the centrifugal force and the force of gravity, and each has an associated potential energy function. As an initial attempt at modeling the motion, we assume that for both the offset atom and the buckyball, the frictional effects are negligible and can be ignored. Essentially, we assume that ultralow friction, as illustrated by Cumings and Zettl ${ }^{3}$ is also applicable here, so that friction is negligible in comparison to the other forces. Note that the plane of the torus is taken to be perpendicular to the direction of gravity so that the angle of inclination is zero.

The van der Waals interaction force is derived from the Lennard-Jones energy, thus $F_{v}=-\nabla V_{1}(x, y, z)$, where $x, y, z$ refer to the coordinates of the offset atom or $\mathrm{C}_{60}$ fullerene, and the three components of the van der Waals force are given by

$$
F_{v_{x}}=-\frac{\partial V_{1}}{\partial x}, F_{v_{y}}=-\frac{\partial V_{1}}{\partial y}, F_{v_{z}}=-\frac{\partial V_{1}}{\partial z} .
$$

As the $\mathrm{C}_{60}$ fullerene orbits around the nanotorus it experiences a centrifugal force. This is the force experienced by a body spinning on an axis and is directed away from the center of rotation. The centrifugal force is $F_{c}=-m R \omega^{2}$, where $m$ is the mass of the rotating body, with corresponding energy $V_{2}=-m R^{2} \omega^{2} / 2$. The $\mathrm{C}_{60}$ fullerene also experiences a gravitational effect as it rotates, defined by $F_{g}=-m g$, where $m$ is the mass of the $\mathrm{C}_{60}$ fullerene and $g$ is the acceleration due to gravity $9.81 \mathrm{~m} / \mathrm{s}^{2}$, with corresponding potential energy $V_{3}=-m g h$, where $h$ is the height above some datum level, and assuming the plane of the nanotorus is positioned in a horizontal plane. Thus, the total energy becomes $V$ $=V_{1}+V_{2}+V_{3}$, and the position of the orbiting atom or buckyball is located where this total energy is minimized. Namely, the three equations arising from Newton's second laws for orbital motion, arise as a consequence of minimizing $V$ [see, for example, Eq. (2.5) below for the atom].

We now give the force balance on a single atom rotating around the nanotorus. The position of the atom is assumed to be located at the point defined by the coordinates $\left(x_{1}, y_{1}, z_{1}\right)$. The forces acting on the body are the van der Waals interaction force, centrifugal force and gravity. Figure 1 illustrates the forces acting on an individual carbon atom, offset a dis- 


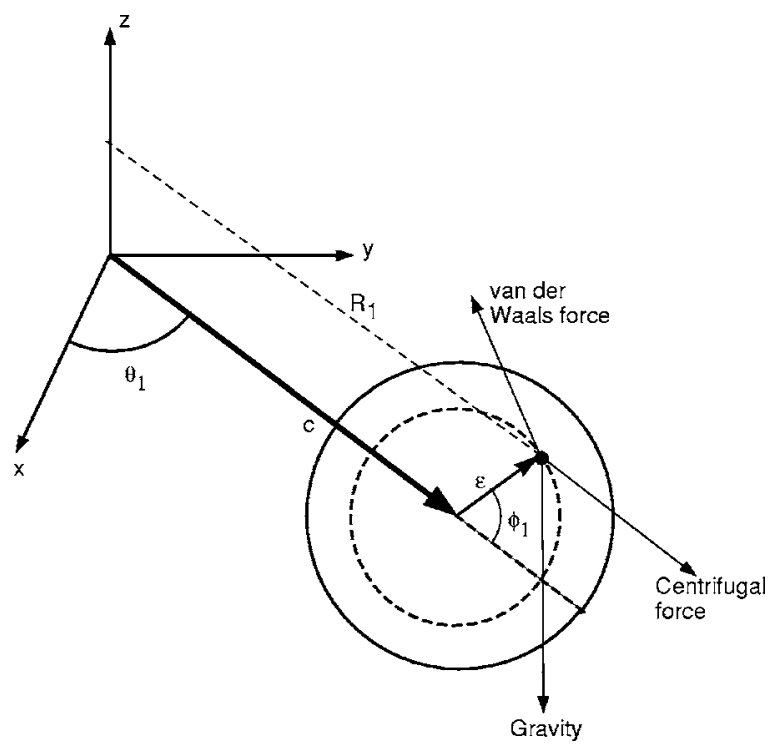

FIG. 1. Forces on an offset carbon atom orbiting inside a nanotorus.

tance $\varepsilon$ from the torus tube center, where $R_{1}=c+\varepsilon \cos \phi_{1}$. In cylindrical polar coordinates $\left(R_{1}, \theta_{1}, z_{1}\right)$, the three equations arising from Newton's second law become

$$
\begin{aligned}
& m\left(\ddot{R}_{1}-R_{1} \dot{\theta}_{1}^{2}\right)=-\frac{\partial V_{1}}{\partial R_{1}}, m\left(R_{1} \ddot{\theta}_{1}+2 \dot{R}_{1} \dot{\theta}_{1}\right)=-\frac{1}{R_{1}} \frac{\partial V_{1}}{\partial \theta_{1}}, \\
& m\left(\ddot{z}_{1}-g\right)=-\frac{\partial V_{1}}{\partial z_{1}},
\end{aligned}
$$

where the dot denotes differentiation with respect to time and $m$ is the assumed mass. Now since we are assuming $R_{1}=c$ $+\varepsilon \cos \phi_{1}$ and $z_{1}=\varepsilon \sin \phi_{1}$ are fixed in space and that the atom is orbiting around the $z$ axis with constant angular velocity $\omega$ (i.e., $\theta_{1}=\omega t$ ), the above three equations become

$$
\frac{\partial V_{1}}{\partial R_{1}}=m R_{1} \omega^{2}, \frac{\partial V_{1}}{\partial \theta_{1}}=0, \frac{\partial V_{1}}{\partial z_{1}}=m g,
$$

where $V_{1}\left(R_{1}, \theta_{1}, z_{1}\right)$ is the Lennard-Jones potential which we detail in Sec. III.

\section{Initial orbiting velocity}

In the present investigation we assume that it is possible to close a $\mathrm{C}_{60}$-nanotube oscillator around onto itself so as to form the $\mathrm{C}_{60}$-nanotorus seamlessly. The vacuum effect, where a $\mathrm{C}_{60}$ fullerene is sucked into one end of the nanotube, ${ }^{6,9}$ generating an initial velocity, is assumed to occur just prior to closure of the nanotorus. The $\mathrm{C}_{60}$-nanotorus oscillator then consists of a fullerene orbiting at an initial velocity equal to the velocity of the $\mathrm{C}_{60}$-nanotube oscillator, found to have a maximum velocity ${ }^{7}$ of $1214 \mathrm{~m} / \mathrm{s}$, approximately $800 \mathrm{~m} / \mathrm{s}$ at suction ${ }^{9}$ and approximately $400 \mathrm{~m} / \mathrm{s}$ steady-state velocity. ${ }^{9}$ This linear velocity may be converted into an equivalent angular velocity $\omega_{0}=v_{0} / R_{1}$, where $v_{0}$ is the initial velocity given above and $R_{1}=c+\varepsilon \cos \phi_{1}$, is the distance from the center of rotation to the center of the rotating body. This value for the velocity is used in the following sections to determine the position of the orbiting body.
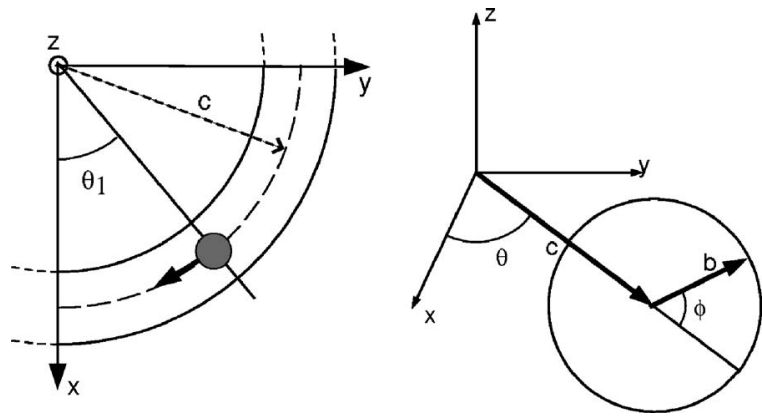

FIG. 2. Torus and its coordinate frame definition.

\section{EQUILIBRIUM OF OFFSET ATOM}

In this section, we determine the Lennard-Jones energy for an offset carbon atom inside a carbon nanotorus and we ascertain the minimum energy (equilibrium) position. Figures 1 and 2 illustrate the offset position, the torus coordinate frame and coordinates used to define a typical point on the torus surface, which in Cartesian coordinates, $(x, y, z)$ is given as

$$
\begin{aligned}
& x=(c+b \cos \phi) \cos \theta, \quad y=(c+b \cos \phi) \sin \theta, \\
& z=b \sin \phi,
\end{aligned}
$$

where $b$ is the nanotorus tube radius and $c$ is the nanotorus ring radius. We fix the location of the offset carbon atom to be a distance $\varepsilon$ from the cross-sectional center of the torus, which in Cartesian coordinates is defined by

$$
\begin{aligned}
& x_{1}=\left(c+\varepsilon \cos \phi_{1}\right) \cos \theta_{1}, \quad y_{1}=\left(c+\varepsilon \cos \phi_{1}\right) \sin \theta_{1}, \\
& z_{1}=\varepsilon \sin \phi_{1} .
\end{aligned}
$$

Using Eqs. (2.2) and (2.3) we calculate the Lennard-Jones energy between the carbon atom and the surface of the torus

$$
V_{1}=\eta_{t} \int_{0}^{2 \pi} \int_{0}^{2 \pi}\left(\frac{-A}{\rho^{6}}+\frac{B}{\rho^{12}}\right) b(c+b \cos \phi) d \phi d \theta,
$$

and the distance between the torus surface element and the carbon atom is

$$
\begin{aligned}
\rho^{2}= & \left(x-x_{1}\right)^{2}+\left(y-y_{1}\right)^{2}+\left(z-z_{1}\right)^{2} \\
= & (b-\varepsilon)^{2}+4(c+b \cos \phi) \\
& \times\left(c+\varepsilon \cos \varphi_{1}\right) \sin ^{2}\left[\left(\theta-\theta_{1}\right) / 2\right] \\
& +4 b \varepsilon \sin ^{2}\left[\left(\phi-\phi_{1}\right) / 2\right] .
\end{aligned}
$$

Details for the evaluation of the integral Eq. (3.1) are given in Appendix A in terms of hypergeometric and Legendre functions. For the atom offset by an amount $\varepsilon$ from the tube center, the resulting Lennard-Jones energy is shown to be given by the elegant result

$$
\begin{aligned}
V_{1}= & \frac{3 \pi^{2} b c^{1 / 2} \eta_{t}}{4(c+\varepsilon)^{1 / 2}}\left[\frac{-A}{\left(b^{2}-\varepsilon^{2}\right)^{5 / 2}} P_{3 / 2}\left(\frac{b^{2}+\varepsilon^{2}}{b^{2}-\varepsilon^{2}}\right)\right. \\
& \left.+\frac{21 B}{32\left(b^{2}-\varepsilon^{2}\right)^{11 / 2}} P_{9 / 2}\left(\frac{b^{2}+\varepsilon^{2}}{b^{2}-\varepsilon^{2}}\right)\right]
\end{aligned}
$$

where $b$ is the tube radius, $c$ is the nanotorus ring radius, 


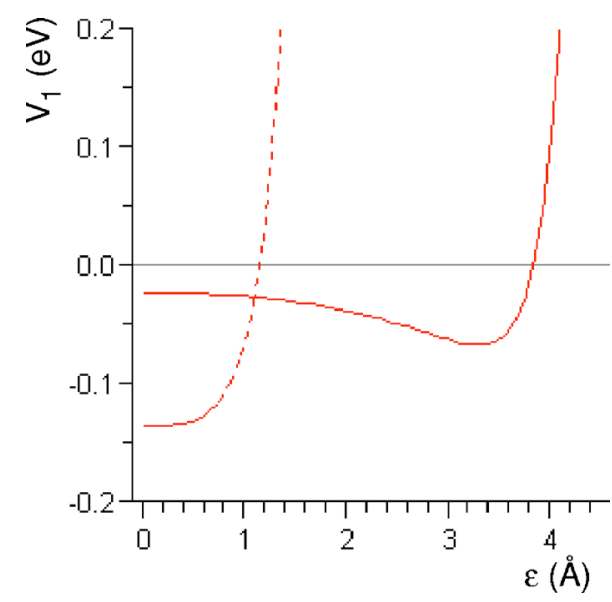

FIG. 3. Lennard-Jones energy $V_{1}$ against atom offset position $\varepsilon$ [dashed (6, 6), solid $(10,10)]$.

$P_{n}(z)$ is the Legendre function of the first kind, and $\eta_{t}$ is the mean surface density of the carbon atoms on the nanotorus. We comment that under the assumption $\varepsilon<b \ll c$, Eq. (3.3) is an approximate formula and constitutes only the leading order terms of an integral involving several such terms and which are fully detailed in Appendix A.

Using the algebraic package, MAPLE, we plot the Lennard-Jones energy $V_{1}$ against the atom offset position $\varepsilon$ as shown in Fig. 3. The equilibrium position is found to vary with the nanotorus tube radius $b$, and the atom moves closer to the tube wall as the radius $b$ increases, where we assume a nanotorus ring radius $c$ of $1500 \AA$ from Liu et al. ${ }^{11}$ For a carbon nanotorus created by closing a $(6,6)$ carbon nanotube ( $b=4.071 \AA$ ) onto itself, we obtain the equilibrium position $\varepsilon=0 \AA$, that is, the equilibrium location lies on the circle $R_{1}=c$. Similarly, for a nanotorus $(10,10)(b=6.784 \AA)$ we obtain an equilibrium position $\varepsilon=3.29 \AA$. These results compare well with Cox, Thamwattana, and $\mathrm{Hill}^{20}$ for the $\mathrm{C}_{60}$-nanotube oscillator. In fact, taking the limit as $c$ tends to infinity gives the identical result for the offset atom of Cox, Thamwattana, and Hill. ${ }^{20}$ Their energy, given in terms of hypergeometric functions, can be transformed into precisely the formula Eq. (3.3).

Figures 4 and 5 illustrate the centrifugal and gravity energies, respectively, plotted against the atom offset position $\varepsilon$. To obtain the initial velocity used in these plots we set $\varepsilon$ $=0$ and calculate the initial velocity from $\omega_{0}=v_{0} / R_{1}$, given in Sec. II C. It is important to note that from these figures the gravity potential which is of the order $10^{-15} \mathrm{eV}$ is negligible in comparison to the centrifugal and Lennard-Jones energies. Under the influence of centrifugal forces alone, the minimum energy position for the offset atom is as far from the center of the nanotorus as is possible, namely $\phi_{1}=0$ and $\varepsilon=b$. This is reasonable as the centrifugal force acts to push an orbiting body away from the center of rotation. The centrifugal potential influences the minimum energy position by a shift away from the nanotorus tube center, observed for angular velocities in the gigahertz range. For example, for the (10, 10) carbon nanotorus, to move the atom $0.5 \AA$ further from the equilibrium position and away from the nanotorus center

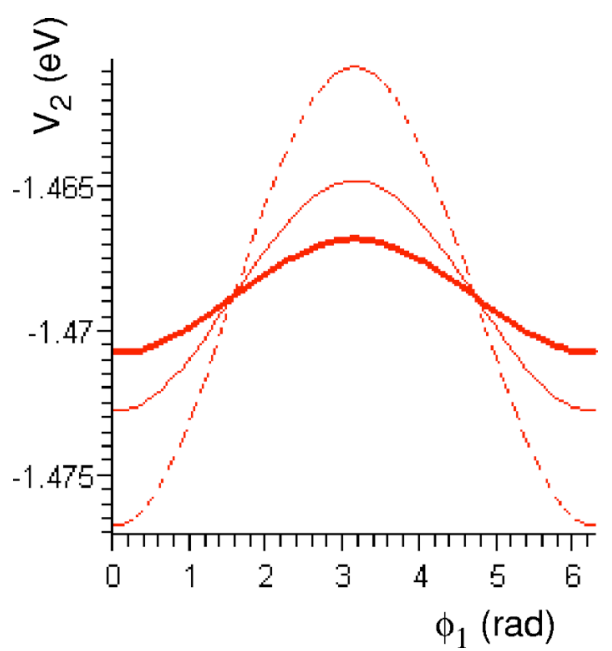

FIG. 4. Centrifugal energy $V_{2}$ against atom offset angle $\phi_{1}$ [dashed $\varepsilon=b$, normal $\varepsilon=b / 2$, bold $\varepsilon=1]$

a frequency of $37 \mathrm{GHz}$ is required. As the angular velocity increases the atom shifts further from the nanotorus tube center.

\section{EQUILIBRIUM OF $\mathrm{C}_{60}$ FULLERENE}

Here, we assume that the $\mathrm{C}_{60}$ fullerene center is located at the position defined by $\left(\varepsilon, \phi_{1}\right)$. We apply the same technique as for the atom except that we must also integrate over the surface of the $\mathrm{C}_{60}$ fullerene. We start by evaluating the interaction potential between the fullerene and an arbitrary surface. The distance from the surface of the fullerene to an arbitrary surface is $\rho^{2}=a^{2}+r^{2}-2 a r \cos \beta$, where $a$ is the $\mathrm{C}_{60}$ fullerene radius, $\beta$ is the angle shown in Fig. 6 , and $r$ is the distance from the center of the fullerene to an arbitrary point on the torus surface. The Lennard-Jones energy for the fullerene is

$$
\begin{aligned}
E_{n}=\int_{0}^{\pi} \frac{1}{\rho^{n}} 2 \pi a^{2} \sin \beta d \beta & =\frac{2 \pi a}{r} \int_{a-r}^{a+r} \rho^{1-n} d \rho \\
& =\frac{2 \pi a}{r}\left[\frac{\rho^{2-n}}{2-n}\right]_{a-r}^{a+r} .
\end{aligned}
$$

Here, we are interested in the two values $n=6$ and $n=12$ so

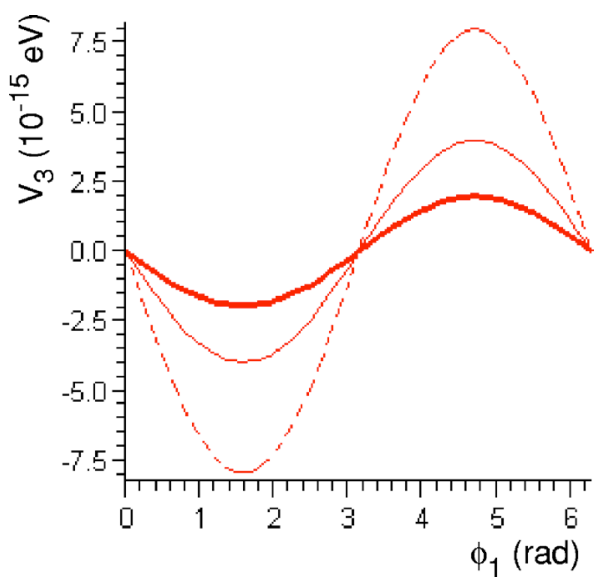

FIG. 5. Gravitational energy $V_{3}$ against atom offset angle $\phi_{1}$ [dashed $\varepsilon=b$, normal $\varepsilon=b / 2$, bold $\varepsilon=1]$. 


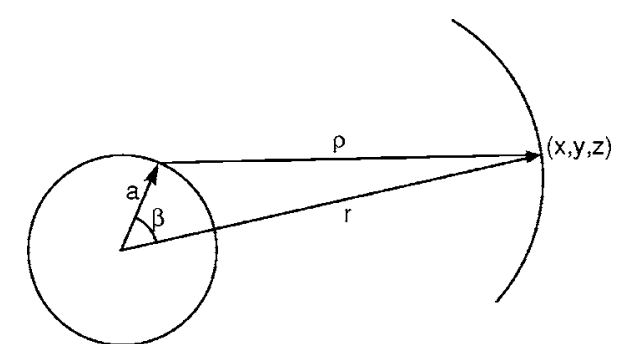

FIG. 6. Fullerene interacting with an arbitrary surface.

that the energy of the buckyball, $E_{\text {bucky }}=-A E_{6}+B E_{12}$ becomes

$$
\begin{aligned}
E_{\text {bucky }}= & \frac{2 \pi a \eta_{b}}{r}\left[\frac{A}{4}\left(\frac{1}{(r+a)^{4}}-\frac{1}{(r-a)^{4}}\right)-\frac{B}{10}\left(\frac{1}{(r+a)^{10}}\right.\right. \\
& \left.\left.-\frac{1}{(r-a)^{10}}\right)\right] .
\end{aligned}
$$

Note that from Appendix B we have

$$
\begin{aligned}
{\left[\rho^{2-n}\right]_{a-r}^{a+r} } & =\left[\frac{1}{\rho^{2 m}}\right]_{a-r}^{a+r} \\
& =\frac{-2}{\left(r^{2}-a^{2}\right)^{2 m}} \sum_{j=0}^{m-1}\left(\begin{array}{c}
2 m \\
2 j+1
\end{array}\right) r^{2 j+1} a^{2 m-2 j+1},
\end{aligned}
$$

where $2 m=n-2$, so that we are interested in the two particular values $m=2$ and $m=5$. We now integrate over the surface of the torus so that we have

$$
\begin{aligned}
K_{2 m}= & \frac{2 \pi a \eta_{b} \eta_{t} b}{m} \int_{0}^{2 \pi} \int_{0}^{2 \pi} \frac{1}{\left(r^{2}-a^{2}\right)^{2 m}} \sum_{j=0}^{m-1}\left(\begin{array}{c}
2 m \\
2 j+1
\end{array}\right) \\
& \times r^{2 j} a^{2 m-2 j+1}(c+b \cos \phi) d \theta d \phi,
\end{aligned}
$$

where the energy is given by $V_{1}=-A K_{4}+B K_{10}$. Details for evaluating Eq. (4.3) are given in Appendix B, and both one and two variable hypergeometric functions are used. For the buckyball with the center located a distance $\varepsilon$ from the tube center, the resulting Lennard-Jones energy is shown to be given approximately by

$$
\begin{aligned}
V_{1}= & 256 \pi^{3} a b c \eta_{b} \eta_{t} \\
& \times\left[-\frac{5 A a^{3} c^{3}(c+\varepsilon)^{3}}{2[\delta(4 c(c+\varepsilon)+\delta)]^{7 / 2}} F_{1}\left(\frac{1}{2} ; \frac{7}{2}, \frac{7}{2} ; 1 ; \alpha, \beta\right)\right. \\
& \left.+\frac{155584 B a^{9} c^{9}(c+\varepsilon)^{9}}{[\delta(4 c(c+\varepsilon)+\delta)]^{19 / 2}} F_{1}\left(\frac{1}{2} ; \frac{19}{2}, \frac{19}{2} ; 1 ; \alpha, \beta\right)\right],
\end{aligned}
$$

where $b$ is the nanotorus tube radius, $c$ is the nanotorus ring radius, $a$ is the buckyball radius, $\delta=(b+\varepsilon)^{2}-a^{2}, \alpha=4 b \varepsilon / \delta$, $\beta=4 b \varepsilon /[4 c(c+\varepsilon)+\delta], \eta_{b}$, and $\eta_{t}$ are the buckyball and nanotorus mean surface densities, respectively. Note that the energy given above is only valid for $|\varepsilon| \leq|b-a|$ and $F_{1}\left(\alpha ; \beta, \beta^{\prime} ; \gamma ; x, y\right)$ is Appell's hypergeometric function of two variables. Again in deriving Eq. (4.4) we have retained only the leading order terms.

Using the algebraic package, MAPLE, we plot the Lennard-Jones energy $V_{1}$ against the buckyball's position $\varepsilon$,

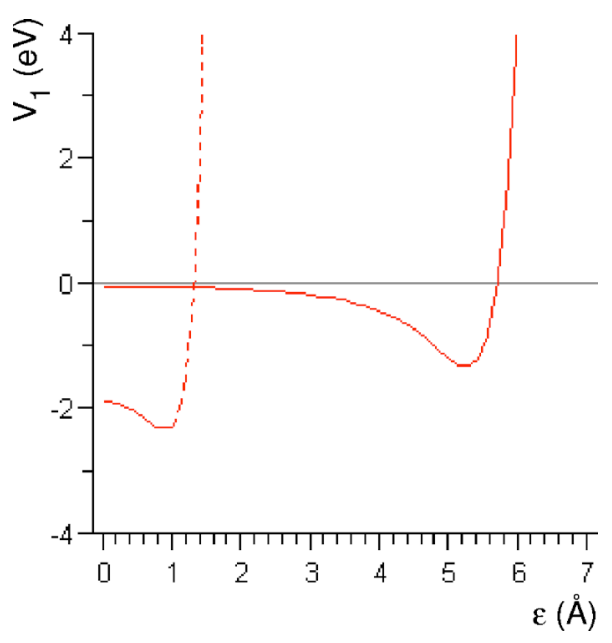

FIG. 7. Lennard-Jones energy $V_{1}$ against buckyball position $\varepsilon$ [dashed (10, $10)$, solid $(16,16)]$.

shown in Fig. 7 for both the nanotorus $(10,10)(b$ $=6.784 \AA)$ and $(16,16)(b=10.856 \AA)$, where $c$ is $1500 \AA$ and $\phi_{1}=0$. As in Sec. III, gravity is again found to be negligible in comparison to the centrifugal and Lennard-Jones energies. The buckyball's minimum energy (equilibrium) position $\varepsilon$ is found to depend on the nanotorus tube radius $b$, and the buckyball moves closer to the nanotorus wall as the radius $b$ increases. For example, for the equilibrium position of a $(10,10)$ nanotorus we obtain $\varepsilon=0.9 \AA$ and for $(16,16)$ we obtain $\varepsilon=5.25 \AA$, representing a distance from the wall of 2.334 and $2.056 \AA$, respectively. A similar observation is made by Cox, Thamwattana, and $\mathrm{Hill}^{20}$ for the $\mathrm{C}_{60}$-nanotube oscillator.

Again, we observe that the centrifugal potential alters the minimum energy position, and the minimum energy position moves further from the center of rotation $\left(\phi_{1}=0\right)$, noting that here we use the same initial velocity to that used in Sec. III. The movement depends on the nanotorus tube radius $b$; for example, the $(10,10)$ nanotorus moves $7 \%$ closer to the tube wall and the $(16,16)$ nanotorus moves $2 \%$ closer to the tube wall. Again, a shift in the minimum energy position is observed for angular velocities in the gigahertz range and this shift increases as the angular velocity increases.

In the limit as $c$ tends to infinity for Eq. (4.4), we may use the result from Colavecchia, Gasaneo, and Miraglia ${ }^{21}$ $F_{1}\left(\alpha ; \beta, \beta_{1} ; \gamma ; x, 0\right)={ }_{2} F_{1}(\alpha, \beta ; \gamma ; x)$, to obtain overall agreement with Cox, Thamwattana, and $\mathrm{Hill}^{20}$ for the $\mathrm{C}_{60}$-nanotube oscillator. However, to obtain an equation for the Lennard-Jones energy of the buckyball, only the leading order contributions were retained and as a consequence there is a difference between the two models, which becomes smaller as the tube radius $b$ increases.

\section{ORBITING VELOCITY}

Using the standard analysis from orbital motion we have that the angular momentum, $L$ for a circular orbit is $L$ $=m \omega R^{2}$, where $\omega$ is the angular velocity and $R$ is the distance from the center of rotation to the center of the rotating particle. Since the angular momentum is conserved for a circular orbit we can infer that $R$ decreases with increasing $\omega$. 


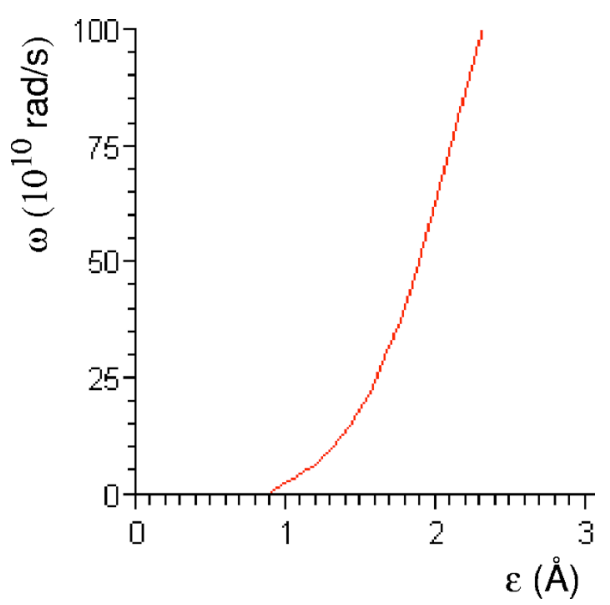

FIG. 8. Angular velocity $\omega$ against buckyball position $\varepsilon$ for $(10,10)$.

In this section we formulate a relationship between the velocity of the orbiting body and the position of the center, $\left(\varepsilon, \phi_{1}\right)$. From the results shown in Sec. III we see that gravity may be neglected and, therefore, the atom may be assumed to be located at $\phi_{1}=0$ as a result of the centrifugal potential. Therefore, the three Eqs. (2.5) reduce to

$$
\frac{\partial V_{1}}{\partial R}=m R \omega^{2},
$$

and the remaining two equations are satisfied identically. Using the above equation where $F(\varepsilon)=\partial V_{1} / \partial R$, we can determine a relationship in the form

$$
\omega^{2}=\frac{F(\varepsilon)}{m(c+\varepsilon)} .
$$

Figure 8 illustrates the angular velocity against the offset position $\varepsilon$ for the $(10,10)$ nanotorus with an orbiting buckyball. As shown, the buckyball will automatically move to its equilibrium position with no applied angular velocity. As a result there is no angular velocity until we reach this position. To move the buckyball from its equilibrium position, away from the cross-sectional center of the nanotorus an angular velocity must be applied. For example, for the $(10,10)$ nanotorus we obtain an equilibrium position $\varepsilon=0.9 \AA$, using the relation $\omega=2 \pi f$ a frequency of $34 \mathrm{GHz}$ would move the buckyball another $0.4 \AA$ closer to the wall or away from its equilibrium position, equivalent to approximately $2 \mathrm{eV}$ of energy, as shown in Fig. 7. We may compare this frequency to that given by Cox, Thamwattana, and Hill, ${ }^{20}$ who obtain a frequency of $36 \mathrm{GHz}$ for the $(10,10) \mathrm{C}_{60}$-nanotube oscillator. The greater the induced frequency the further the buckyball moves from the center of the nanotorus. For example, a frequency of $150 \mathrm{GHz}$ moves the buckyball $1 \AA$ away from the equilibrium position. As shown in Fig. 8 the velocity increases exponentially as the distance $\varepsilon$ increases. The frequencies that are required to shift the buckyball from the equilibrium position for the $\mathrm{C}_{60}$-nanotorus are in the gigahertz range. Clearly by adjustment of the orbiting location of the buckyball, the $\mathrm{C}_{60}$-nanotorus orbiter has the capacity to produce a wide frequency range as high as $150 \mathrm{GHz}$ with no fundamental change in structural geometry.

\section{CONCLUSIONS}

Following Cumings and Zettl, ${ }^{3}$ and as an initial attempt to model a $\mathrm{C}_{60}$-nanotorus orbiter we ignore any frictional effects. Although we consider the effect of gravity, we show that for a horizontal nanotorus, the effects of gravity are considerably less than those arising from the Lennard-Jones energy and the centrifugal forces.

For a carbon nanotorus containing an offset atom, the equilibrium position is found to depend on the nanotorus tube radius $b$, and the atom is shown to move closer to the tube wall as this radius increases. For example, for a carbon nanotorus created from closing a $(6,6)$ carbon nanotube $(b$ $=4.071 \AA$ ) onto itself, we obtain an equilibrium position $\varepsilon$ $=0 \AA$. However, for a nanotorus $(10,10)(b=6.784 \AA)$ we obtain $\varepsilon=3.29 \AA$. These results compare very well with the corresponding results of Cox, Thamwattana, and Hill ${ }^{20}$ for the $\mathrm{C}_{60}$-nanotube oscillator. In fact, in the limit as $c$ tends to infinity our Eq. (3.3) gives precisely the same formula as that given by Cox, Thamwattana, and Hill. ${ }^{20}$ The centrifugal effect is found to shift the minimum energy position of the atom away from the center of rotation at frequencies in the gigahertz range.

Similarly, the equilibrium position for the buckyball is found to depend on the nanotorus tube radius $b$, and again the buckyball moves closer to the tube wall as the radius $b$ increases. For example, for a carbon nanotorus created from closing a $(10,10)$ carbon nanotube $(b=6.784 \AA)$ we obtain an equilibrium distance $\varepsilon=0.9 \AA$, while for a nanotorus (16, 16) $(b=10.856 \AA)$ we obtain $\varepsilon=5.25 \AA$. Again, a similar observation is made by Cox, Thamwattana, and Hill. ${ }^{20}$ Assuming that the angle $\varphi_{1}$ is zero, the inclusion of centrifugal energy affects the buckyball's minimum energy position, causing the fullerene to move further from the center of the nanotorus (center of rotation) and hence closer to the tube wall. This effect varies with the nanotorus tube radius $b$, and is more prominent as the angular velocity increases, and is observed for frequencies in the gigahertz range. In the limit as $c$ tends to infinity, Eq. (4.4) gives overall agreement with the results given by Cox, Thamwattana, and $\mathrm{Hill}^{20}$ for the $\mathrm{C}_{60}$-nanotube oscillator. However, for the nanotorus, in order to obtain Eq. (4.4) for the Lennard-Jones energy of the buckyball, only leading order contributions were retained and as a consequence there is a small difference between the two models, which decreases as the tube radius $b$ increases.

For any prescribed angular velocity we may determine the equilibrium position $\varepsilon$ of the $\mathrm{C}_{60}$ fullerene. Alternatively, for given $\varepsilon$ the angular velocity may be determined. Frequencies in the gigahertz range are required to shift the orbiting body away from its equilibrium position. For example, the $(10,10)$ carbon nanotorus, a frequency of $34 \mathrm{GHz}$ is required to move the buckyball $0.4 \AA$ and a frequency of 150 $\mathrm{GHz}$ is required to move $1 \AA$. Thus, by adjustment of the equilibrium location the $\mathrm{C}_{60}$-nanotorus orbiter may provide a wide range of frequencies for no fundamental change in geometric structure. We note that for the $(10,10)$ carbon nanotube Cox, Thamwattana, and Hill ${ }^{20}$ find a frequency of 36 $\mathrm{GHz}$ for the $\mathrm{C}_{60}$-nanotube oscillator. 
In summary, the key findings of the analysis presented here are:

- The effect of gravity is negligible;

- centrifugal forces affect the position of both the offset atom and the fullerene, shifting their minimum energy position away from the center of the nanotorus ring;

- the minimum energy position of both the offset atom and the buckyball depend on the nanotorus tube radius $b$;

- for both the energy and the location of both the offset atom and the $\mathrm{C}_{60}$ fullerene, the model agrees with corresponding results from Cox, Thamwattana, and $\mathrm{Hill}^{20}$ in the limit as $c$ tends to infinity;

- frequencies are obtained in the gigahertz range, as high as $150 \mathrm{GHz}$, and may possibly be controlled by changing the orbiting position $\varepsilon$.

We conclude by commenting that although the $\mathrm{C}_{60}$-nanotorus orbiter is speculative in nature, and presents exciting possibilities, there are still many practical challenges that would need to be overcome before the $\mathrm{C}_{60}$-nanotorus orbiter might be realized. However, the present theoretical study is a necessary precursor to any such developments.

\section{ACKNOWLEDGMENTS}

The authors are grateful to the Australian Research Council for support through the Discovery Project Scheme and the University of Wollongong for a University Postgraduate Award. The authors also wish to acknowledge Barry Cox, Duangkamon Baowan, and Ngamta Thamwattana for many helpful comments and discussions.

\section{APPENDIX A: EVALUATION OF INTEGRALS FOR THE OFFSET ATOM}

In this Appendix we evaluate the various integrals for the energy for an offset atom inside a nanotorus. In the first section we perform the $\theta$ integration for the integrals $I_{n}$ with $n$ being any positive integer. The section thereafter performs the $\varphi$ integration.

\section{Evaluation of $\theta$ integration}

In this Appendix for arbitrary $n$ we evaluate the integrals $I_{n}$ defined by

$$
I_{n}=\int_{0}^{2 \pi} \frac{d \theta}{\rho^{n}}
$$

where $\rho$ is defined by Eq. (3.2). We write Eq. (3.2) as

$$
\rho=\sqrt{N(\phi)+M(\phi) \sin ^{2}\left[\left(\theta-\theta_{1}\right) / 2\right]},
$$

where $M$ and $N$ are defined by

$$
\begin{aligned}
& M(\phi)=4(c+b \cos \phi)\left(c+\varepsilon \cos \phi_{1}\right), \\
& N(\phi)=(b-\varepsilon)^{2}+4 \varepsilon b \sin ^{2}\left[\left(\phi-\phi_{1}\right) / 2\right] .
\end{aligned}
$$

The Lennard-Jones energy involves integrals of the form

$$
I_{n}\left(\varepsilon, \theta_{1}, \phi_{1}\right)=\int_{0}^{2 \pi} \int_{0}^{2 \pi} \frac{b(c+b \cos \phi)}{\rho^{n}} d \theta d \phi
$$

and it is a simple matter to show that $\partial I_{n} / \partial \theta_{1}$ is automatically zero as follows. From Eq. (A2) we have on formal differentiation with respect to $\theta_{1}$

$$
\begin{aligned}
\frac{\partial I_{n}}{\partial \theta_{1}}= & \int_{0}^{2 \pi} \int_{0}^{2 \pi} \frac{n b(c+b \cos \phi)}{2 \rho^{n+2}} \\
& \times M \cos \left[\left(\theta-\theta_{1}\right) / 2\right] \sin \left[\left(\theta-\theta_{1}\right) / 2\right] d \theta d \phi \\
= & -\int_{0}^{2 \pi} b(c+b \cos \phi)\left[\frac{1}{\rho^{n}}\right]_{0}^{2 \pi} d \phi,
\end{aligned}
$$

and since $\sin \left(2 \pi-\theta_{1}\right) / 2=\sin \left(\theta_{1} / 2\right)$, it follows that the integrand is identically zero. The result $\partial I_{n} / \partial \theta_{1}=0$ implies that we may evaluate the integral Eq. (A2) for any convenient value of $\theta_{1}$, say $\theta_{1}=0$. Thus Eq. (A2) becomes

$$
I_{n}\left(\varepsilon, \phi_{1}\right)=\int_{0}^{2 \pi} \int_{0}^{2 \pi} \frac{b(c+b \cos \phi)}{\rho^{n}} d \theta d \phi,
$$

where now $\rho$ is defined by $\rho=\sqrt{N+M \sin ^{2}(\theta / 2)}$. We now let $x=\theta / 2$ so that we have

$$
I_{n}=2 \int_{0}^{\pi} \frac{d x}{\rho^{n}}=2\left(\int_{0}^{\pi / 2} \frac{d x}{\rho^{n}}+\int_{\pi / 2}^{\pi} \frac{d x}{\rho^{n}}\right) .
$$

We now make the substitution $y=\pi-x$ for the integral from $[\pi / 2, \pi]$ so that Eq. (A3) becomes

$$
\begin{aligned}
I_{n} & =4 \int_{0}^{\pi / 2} \frac{d x}{\rho^{n}}=4 \int_{0}^{\pi / 2} \frac{d x}{\left(N+M \sin ^{2} x\right)^{n / 2}} \\
& =4 \int_{0}^{\pi / 2} \frac{d x}{\left(N+M \sin ^{2} x\right)^{m}},
\end{aligned}
$$

where $n=2 m$ and we are interested in the two values $m=3$ and $m=6$. On making the further substitution $t=\cot x$ we obtain

$$
\begin{aligned}
I_{2 m} & =4 \int_{0}^{\pi / 2} \frac{\csc ^{2 m-2} x \csc ^{2} x}{\left(N \csc ^{2} x+M\right)^{m}} d x \\
& =-4 \int_{0}^{\pi / 2} \frac{\left(\csc ^{2} x\right)^{m-1}}{\left(N \csc ^{2} x+M\right)^{m}} d(\cot x) \\
& =4 \int_{0}^{\infty} \frac{\left(t^{2}+1\right)^{m-1}}{\left(N t^{2}+N+M\right)^{m}} d t \\
& =\frac{4}{(N+M)^{m}} \int_{0}^{\infty} \frac{\left(t^{2}+1\right)^{m-1}}{\left(\gamma t^{2}+1\right)^{m}} d t,
\end{aligned}
$$

where $\gamma=N /(N+M)$. Now on writing this integral in the form

$$
I_{2 m}=\frac{4}{(N+M)^{m}} \int_{0}^{\infty} \frac{1}{\left[1+(\gamma-1) t^{2} /\left(1+t^{2}\right)\right]^{m}} \frac{d t}{\left(t^{2}+1\right)},
$$

we are led to make the substitution 


$$
z=\frac{t}{\left(1+t^{2}\right)^{1 / 2}}, \quad t=\frac{z}{\left(1-z^{2}\right)^{1 / 2}}, \quad d t=\frac{d z}{\left(1-z^{2}\right)^{3 / 2}},
$$

to obtain

$$
\begin{aligned}
I_{2 m} & =\frac{4}{(N+M)^{m}} \int_{0}^{1} \frac{d z}{\left[1+(1-\gamma) z^{2}\right]^{m}\left(1-z^{2}\right)^{1 / 2}} \\
& =\frac{2}{(N+M)^{m}} \int_{0}^{1} \frac{u^{1 / 2}(1-u)^{-1 / 2}}{[1-(1-\gamma) u]^{m}} d u,
\end{aligned}
$$

where the final line follows on making the substitution $u$ $=z^{2}$. From Gradshteyn and Ryzhik ${ }^{22}$ we have

$$
I_{2 m}=\frac{2 \pi}{(N+M)^{m}} F\left(m, \frac{1}{2} ; 1 ; 1-\gamma\right),
$$

where $F(a, b ; c ; z)$ denotes the usual hypergeometric function and we have used $\Gamma(1 / 2)=\sqrt{\pi}$. From Gradshteyn and Ryzhik $^{22}$ we have

$$
F(a, b ; c ;-z)=\frac{1}{(1+z)^{a}} F\left(a, c-b ; c ; \frac{z}{1+z}\right),
$$

where $a=m, b=1 / 2, c=1$, and $z=M / N$, we may deduce from Eq. (A5)

$$
I_{2 m}=\frac{2 \pi}{N^{m}} F\left(m, \frac{1}{2} ; 1 ;-\frac{M}{N}\right) .
$$

Thus the integral Eq. (A3) becomes

$$
I_{2 m}\left(\varepsilon, \phi_{1}\right)=2 \pi b \int_{0}^{2 \pi} \frac{F\left(m, \frac{1}{2} ; 1 ;-\frac{M}{N}\right)}{N^{m}}(c+b \cos \phi) d \phi .
$$

From Erdélyi et $a .^{23}$ the hypergeometric function appearing in this integrand can be shown to simplify to become

$$
F\left(m, \frac{1}{2} ; 1 ; z\right)=\sum_{k=0}^{\infty}\left(\begin{array}{c}
m+k-1 \\
k
\end{array}\right)\left(\begin{array}{c}
2 k \\
k
\end{array}\right)\left(\frac{z}{4}\right)^{k} .
$$

Using the algebraic package MAPLE we find that in our case $F(m, 1 / 2 ; 1 ;-z)$ for the two values $m=3$ and $m=6$ become simply

$$
\begin{aligned}
V_{1}= & \eta_{t} \int_{0}^{2 \pi}\left(\frac{-3 A \pi}{4 N^{5 / 2} \sqrt{M}}+\frac{63 B \pi}{128 N^{11 / 2} \sqrt{M}}\right) b(c+b \cos \phi) d \phi \\
= & \frac{3 \eta_{t} \pi}{4} \int_{0}^{2 \pi}\left(\frac{-A}{\left[(b-\varepsilon)^{2}+4 \varepsilon b \sin ^{2}\left(\left(\phi-\phi_{1}\right) / 2\right)\right]^{5 / 2}\left[4(c+b \cos \phi)\left(c+\varepsilon \cos \phi_{1}\right)\right]^{1 / 2}}\right. \\
& \left.+\frac{21 B}{32\left[(b-\varepsilon)^{2}+4 \varepsilon b \sin ^{2}\left(\left(\phi-\phi_{1}\right) / 2\right)\right]^{11 / 2}\left[4(c+b \cos \phi)\left(c+\varepsilon \cos \phi_{1}\right)\right]^{1 / 2}}\right) \times b(c+b \cos \phi) d \phi
\end{aligned}
$$

where $z=M / N$. By a comparison of the relative size of $\varepsilon, b$, and $c$ assuming $\varepsilon<b \ll c$, the dominant terms of the above integral simplify to give where $z=M / N$. Alternatively, this result can be found from Erdélyi et al. $^{23}$ by recognizing that the above hypergeometric function admits a quadratic transformation and is degenerate since one of either $a, b, c-a$, or $c-b$ is an integer. Using the transformation $F(a, b ; c ;-z)=(1+z)^{c-a-b} F(c-a, c-b ; c ;-z)$ the hypergeometric function becomes

$$
\begin{aligned}
F\left(m, \frac{1}{2} ; 1 ;-z\right) & =(1+z)^{1 / 2-m} F\left(1-m, \frac{1}{2} ; 1 ;-z\right) \\
& =(1+z)^{1 / 2-m} \sum_{k=0}^{m-1} \frac{(1-m)_{k}\left(\frac{1}{2}\right)_{k}}{(1)_{k}} \frac{(-z)^{k}}{k !},
\end{aligned}
$$

where $z=M / N$. This agrees with the result provided by MAPLE.

\section{Evaluation of $\phi$ integration}

Using Eq. (A6) the integral for $\phi$ becomes

$$
\begin{aligned}
V_{1}= & \eta_{t} \int_{0}^{2 \pi}\left(-\frac{A \pi}{4 N^{3}} \frac{3 z^{2}+8 z+8}{(z+1)^{5 / 2}}\right. \\
& \left.+\frac{B \pi}{128 N^{6}} \frac{63 z^{5}+350 z^{4}+800 z^{3}+960 z^{2}+640 z+256}{(z+1)^{11 / 2}}\right) \\
& \times b(c+b \cos \phi) d \phi,
\end{aligned}
$$

$F\left(3, \frac{1}{2} ; 1 ;-z\right)=\frac{3 z^{2}+8 z+8}{8(z+1)^{5 / 2}}$,

$$
\begin{gathered}
F\left(6, \frac{1}{2} ; 1 ;-z\right) \\
\quad=\frac{63 z^{5}+350 z^{4}+800 z^{3}+960 z^{2}+640 z+256}{256(z+1)^{3 / 2}\left(z^{4}+4 z^{3}+6 z^{2}+4 z+1\right)},
\end{gathered}
$$

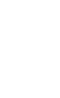


that the radius $c$ is dominant when compared to $b \cos \phi$ and so the integral becomes

$$
\begin{aligned}
V_{1}= & \frac{3 \pi b \eta_{t} c^{1 / 2}}{4 \Sigma^{1 / 2}} \int_{0}^{2 \pi}\left(\frac{-A}{\left[\sigma+\gamma \sin ^{2}\left(\left(\phi-\phi_{1}\right) / 2\right)\right]^{5 / 2}}\right. \\
& \left.+\frac{21 B}{32\left[\sigma+\gamma \sin ^{2}\left(\left(\phi-\phi_{1}\right) / 2\right)\right]^{11 / 2}}\right) d \phi .
\end{aligned}
$$

We know that gravity is negligible compared to the centrifugal and the Lennard-Jones potentials, so that the atom will locate itself in the plane of rotation so as to maximize the centrifugal effect and, therefore, $\phi_{1}=0$. We change the limits of integration, letting $x=\phi / 2$ so that we now have

$$
\begin{aligned}
V_{1}= & \frac{3 \pi b c^{1 / 2} \eta_{t}}{2 \Sigma^{1 / 2}} \int_{0}^{\pi}\left(\frac{-A}{\left(\sigma+\gamma \sin ^{2} x\right)^{5 / 2}}\right. \\
& \left.+\frac{21 B}{32\left(\sigma+\gamma \sin ^{2} x\right)^{11 / 2}}\right) d x,
\end{aligned}
$$

and we can evaluate the integrals for arbitrary $n$, thus

$$
H_{n}=\int_{0}^{\pi} \frac{d x}{\left(\sigma+\gamma \sin ^{2} x\right)^{n / 2}}=2 \int_{0}^{\pi / 2} \frac{d x}{\left(\sigma+\gamma \sin ^{2} x\right)^{m}},
$$

where $n=2 m$ and here we are interested in the two values $m=5 / 2$ and $m=11 / 2$. As above we may deduce

$$
H_{2 m}=\frac{\pi}{(\sigma+\gamma)^{m}} F\left(m, \frac{1}{2} ; 1 ; 1-\beta\right),
$$

where $F(a, b ; c ; z)$ denotes the usual hypergeometric function and we have used $\Gamma(1 / 2)=\sqrt{\pi}$. Again from Gradshteyn and Ryzhik $^{22}$, this can be written in the form

$$
H_{2 m}=\frac{\pi}{\sigma^{m}(1+z)^{m}} F\left(m, \frac{1}{2} ; 1 ; \frac{z}{1+z}\right)=\frac{\pi}{\sigma^{m}} F\left(m, \frac{1}{2} ; 1 ;-z\right),
$$

where $z=\gamma / \sigma$. From Erdélyi et al. ${ }^{23}$ and on recognizing two of the numbers $1-c, \pm(a-b)$, and $\pm(c-a-b)$ are equal to each other it can be shown that this result admits a quadratic transformation and is a Legendre function. Using the quadratic transformation

$$
\begin{aligned}
& F\left(a, b ; 2 b ; 4 z /(1+z)^{2}\right) \\
& \quad=(1+z)^{2 a} F\left(a, a+\frac{1}{2}-b ; b+\frac{1}{2} ; z^{2}\right),
\end{aligned}
$$

we obtain

$$
H_{2 m}=\frac{\pi}{\sigma^{m}}(1+\xi)^{2 m} F\left(m, m ; 1 ; \xi^{2}\right)
$$

where $-z=4 \xi /(1+\xi)^{2}$ and $z=\gamma / \sigma$, so that $\xi=-\varepsilon / b$. Using the definitions from Gradshteyn and Ryzhik ${ }^{22}$

$$
\begin{aligned}
P_{\nu}^{\mu}(z)= & \frac{1}{\Gamma(1-\mu)}\left(\frac{z-1}{z+1}\right)^{-\mu / 2}\left(\frac{z+1}{2}\right)^{\nu} \\
& \times F\left(-\nu,-\nu-\mu ; 1-\mu ; \frac{z-1}{z+1}\right)
\end{aligned}
$$

and

$$
F(a, b ; c ; z)=(1-z)^{c-a-b} F(c-a, c-b ; c ; z),
$$

where $P_{\nu}^{\mu}(z)$ is a Legendre function of the first kind and in our case $\mu$ is zero, we obtain the integral in terms of the Legendre function given by

$$
H_{2 m}=\frac{\pi(1+\xi)^{2 m}}{\sigma^{m}\left(1-\xi^{2}\right)^{m}} P_{m-1}\left(\frac{1+\xi^{2}}{1-\xi^{2}}\right)
$$

where we are interested in the two values $m=5 / 2$ and $m$ $=11 / 2$. So that

$$
V_{1}=\frac{3 \pi r c^{1 / 2} \eta_{t}}{2 \Sigma^{1 / 2}}\left(-A H_{5}+\frac{21 B H_{11}}{32}\right) \text {. }
$$

For the offset atom the resulting Lennard-Jones energy is given by

$$
\begin{aligned}
V_{1}= & \frac{3 \pi b c^{1 / 2} \eta_{t}}{2 \Sigma^{1 / 2}}\left[\frac{-A \pi(1+\xi)^{5}}{\sigma^{5 / 2}\left(1-\xi^{2}\right)^{5 / 2}} P_{3 / 2}\left(\frac{1+\xi^{2}}{1-\xi^{2}}\right)\right. \\
& \left.+\frac{21 B \pi(1+\xi)^{11}}{32 \sigma^{11 / 2}\left(1-\xi^{2}\right)^{11 / 2}} P_{9 / 2}\left(\frac{1+\xi^{2}}{1-\xi^{2}}\right)\right],
\end{aligned}
$$

where $\Sigma=4(c+\varepsilon), \sigma=(b-\varepsilon)^{2}$, and $\xi=-\varepsilon / b$. This can be simplified to

$$
\begin{aligned}
V_{1}= & \frac{3 \pi^{2} b c^{1 / 2} \eta_{t}}{4(c+\varepsilon)^{1 / 2}}\left[\frac{-A}{\left(b^{2}-\varepsilon^{2}\right)^{5 / 2}} P_{5 / 2}\left(\frac{b^{2}+\varepsilon^{2}}{b^{2}-\varepsilon^{2}}\right)\right. \\
& \left.+\frac{21 B}{32\left(b^{2}-\varepsilon^{2}\right)^{11 / 2}} P_{9 / 2}\left(\frac{b^{2}+\varepsilon^{2}}{b^{2}-\varepsilon^{2}}\right)\right] .
\end{aligned}
$$

\section{APPENDIX B: EVALUATION OF INTEGRALS FOR FULLERENE}

In this Appendix we evaluate the integrals arising from the interaction between a $\mathrm{C}_{60}$ fullerene and a nanotorus. First we write the energy for the buckyball in terms of a summation and then in Appendix B 1 we perform the $\theta$ integration which results in hypergeometric functions. The following section (Appendix B 2) performs the $\varphi$ integration, resulting in Appell's hypergeometric functions of two variables.

From Eq. (4.1) we see that the energy for the buckyball is

$$
E_{n}=\frac{2 \pi a \eta_{b}}{r(2-n)}\left(\frac{1}{(r+a)^{n-2}}-\frac{1}{(r-a)^{n-2}}\right),
$$

where $a$ is the radius of the buckyball, $r$ is the distance from the center of the buckyball to an arbitrary surface defined by Eq. (3.2). We are interested in the two values $n=6$ and $n$ $=12$, so that $E_{\text {bucky }}=-A E_{6}+B E_{12}$. Rearranging and using the binomial expansion this can be written

$$
\begin{aligned}
E_{n}= & \frac{-2 \pi a \eta_{b}}{r(n-2)}\left(\frac{(r-a)^{n-2}-(r+a)^{n-2}}{\left(r^{2}-a^{2}\right)^{n-2}}\right) \\
= & \frac{-2 \pi a \eta_{\sigma}}{r p} \frac{1}{\left(r^{2}-a^{2}\right)^{p}}\left[\sum_{k=0}^{p}\left(\begin{array}{l}
p \\
k
\end{array}\right)\right. \\
& \left.\times r^{k}(-a)^{p-k}-\sum_{k=0}^{p}\left(\begin{array}{l}
p \\
k
\end{array}\right) r^{k} a^{p-k}\right],
\end{aligned}
$$


where $p=n-2$. We note that when $p-k$ is even the energy becomes identically zero. The two summation terms add when $p-k$ is odd, therefore $p-k=2 j+1$ and we have

$$
E_{2 m}=\frac{2 \pi a \eta_{b}}{r m} \frac{1}{\left(r^{2}-a^{2}\right)^{2 m}} \sum_{j=0}^{m-1}\left(\begin{array}{c}
2 m \\
2 j+1
\end{array}\right) r^{2 m-2 j-1} a^{2 j+1},
$$

where $p=2 m$ and so $2 m=n-2$. The total energy of the buckyball inside the torus is given by

$$
\begin{aligned}
K_{2 m}= & \frac{2 \pi a \eta_{b} \eta_{t} b}{m} \int_{0}^{2 \pi} \int_{0}^{2 \pi} \frac{1}{r\left(r^{2}-a^{2}\right)^{2 m}} \sum_{j=0}^{m-1}\left(\begin{array}{c}
2 m \\
2 j+1
\end{array}\right) \\
& \times r^{2 m-2 j-1} a^{2 j+1}(c+b \cos \phi) d \theta d \phi,
\end{aligned}
$$

where we are interested in $m=2$ and $m=5$, so that $V_{1}=$ $-A K_{4}+B K_{10}$.

\section{Evaluation of $\theta$ integration}

By expanding the integral, Eq. (4.3) for the two specific values of $m=2$ and $m=5$ the energy for the buckyball inside the nanotorus is

$$
\begin{aligned}
V_{1}= & 2 \pi a \eta_{b} \eta_{t} b \int_{0}^{2 \pi} \int_{0}^{2 \pi}\left[\frac{-8 A a\left(r^{2}+a^{2}\right)}{4\left(r^{2}-a^{2}\right)^{4}}\right. \\
& \left.+\frac{4 B a\left(5 r^{4}+10 r^{2} a^{2}+a^{4}\right)\left(r^{4}+10 r^{2} a^{2}+5 a^{4}\right)}{10\left(r^{2}-a^{2}\right)^{10}}\right] \\
& \times(c+b \cos \phi) d \theta d \phi .
\end{aligned}
$$

Splitting the above integral we can integrate the first term

$$
\begin{aligned}
J_{1} & =\int_{0}^{2 \pi} \frac{r^{2}+a^{2}}{\left(r^{2}-a^{2}\right)^{4}} d \theta \\
& =4 \int_{0}^{\pi / 2} \frac{r^{2}-a^{2}+2 a^{2}}{\left(r^{2}-a^{2}\right)^{4}} d x \\
& =4 \int_{0}^{\pi / 2}\left[\frac{1}{\left(r^{2}-a^{2}\right)^{3}}+\frac{2 a^{2}}{\left(r^{2}-a^{2}\right)^{4}}\right] d x,
\end{aligned}
$$

where we let $x=\theta / 2$ and use partial fractions. Again we may evaluate these integrals using hypergeometric functions for arbitrary $n$ where in the above $n=3$ and $n=4$, and

$$
\begin{aligned}
J_{n}=4 \int_{0}^{\pi / 2} \frac{d x}{\left(r^{2}-a^{2}\right)^{n}} & =4 \int_{0}^{\pi / 2} \frac{d x}{\left(M \sin ^{2} x+N-a^{2}\right)^{n}} \\
& =\frac{2 \pi}{\alpha^{n}} F\left(n, \frac{1}{2} ; 1 ;-z\right),
\end{aligned}
$$

where we let $u=\cot x, \alpha=N-a^{2}$, and $\gamma=\alpha /(M+\alpha)$ and again $M=4(c+b \cos \phi)\left(c+\varepsilon \cos \phi_{1}\right), \quad N=(b-\varepsilon)^{2}+4 b \varepsilon \sin ^{2}[(\phi$ $\left.\left.-\phi_{1}\right) / 2\right]$, and the original integral becomes

$$
J_{1}=\frac{2 \pi}{\alpha^{3}} F\left(2, \frac{1}{2} ; 1 ;-z\right)+\frac{4 \pi a^{2}}{\alpha^{4}} F\left(4, \frac{1}{2} ; 1 ;-z\right),
$$

where $z=M / \alpha$. We note that these are also degenerate since from Erdélyi et $a .^{23}$ either of $a, b, c-a, c-b$ in $F(a, b ; c ; z)$ are integers and using Eq. (A7) we can find the finite series. We now examine the second part of Eq. (B1) making the same substitution $x=\theta / 2$,

$$
\begin{aligned}
J_{2} & =\int_{0}^{2 \pi} \frac{\left(5 r^{4}+10 r^{2} a^{2}+a^{4}\right)\left(r^{4}+10 r^{2} a^{2}+5 a^{4}\right) d \theta}{\left(r^{2}-a^{2}\right)^{10}} \\
& =4 \int_{0}^{\pi / 2} \frac{\left(5 r^{4}+10 r^{2} a^{2}+a^{4}\right)\left(r^{4}+10 r^{2} a^{2}+5 a^{4}\right) d x}{\left(r^{2}-a^{2}\right)^{10}} .
\end{aligned}
$$

Using partial fractions this becomes

$$
\begin{aligned}
J_{2}= & 4 \int_{0}^{\pi / 2}\left[\frac{5}{\left(r^{2}-a^{2}\right)^{6}}+\frac{80 a^{2}}{\left(r^{2}-a^{2}\right)^{7}}+\frac{336 a^{4}}{\left(r^{2}-a^{2}\right)^{8}}\right. \\
& \left.+\frac{512 a^{6}}{\left(r^{2}-a^{2}\right)^{9}}+\frac{256 a^{8}}{\left(r^{2}-a^{2}\right)^{10}}\right] d x,
\end{aligned}
$$

so that we can use the same result from the first integration for $J_{n}$, Eq. (B2) where here $n=6,7,8,9,10$, and $J_{2}$ now becomes

$$
\begin{aligned}
J_{2}= & \frac{10 \pi}{\alpha^{6}} F\left(6, \frac{1}{2} ; 1 ;-z\right)+\frac{160 \pi a^{2}}{\alpha^{7}} F\left(7, \frac{1}{2} ; 1 ;-z\right) \\
& +\frac{672 \pi a^{4}}{\alpha^{8}} F\left(8, \frac{1}{2} ; 1 ;-z\right)+\frac{1024 \pi a^{6}}{\alpha^{9}} F\left(9, \frac{1}{2} ; 1 ;-z\right) \\
& +\frac{512 \pi a^{8}}{\alpha^{10}} F\left(10, \frac{1}{2} ; 1 ;-z\right) .
\end{aligned}
$$

Again we note that these are degenerate hypergeometric functions from Erdélyi et $a l^{23}$ and using Eq. (A7) we can find a finite series.

\section{Evaluation of $\phi$ integration}

The integral for $\phi$ then becomes

$$
V_{1}=2 \pi a \eta_{b} \eta_{t} b \int_{0}^{2 \pi}\left(-2 A a J_{1}+\frac{2}{5} B a J_{2}\right)(c+b \cos \phi) d \phi,
$$

where $J_{1}$ and $J_{2}$ are defined in the previous section as hypergeometric functions and $z=M /\left(N-a^{2}\right)$. By a comparison of the relative size of $\varepsilon, b$, and $c$ assuming $\varepsilon<b \ll c$, and retaining only the leading order terms the above integral can be simplified to

$$
\begin{aligned}
V_{1}= & 2 \pi a b c \eta_{b} \eta_{t} \int_{0}^{2 \pi}\left[\frac{-10 A a^{3} \lambda^{3}}{4 \delta^{7}\left(\lambda+\delta^{2}\right)^{7 / 2}}\right. \\
& \left.+\frac{2431 B a^{9} \lambda^{9}}{64 \delta^{19}\left(\lambda+\delta^{2}\right)^{19 / 2}}\right] d \phi,
\end{aligned}
$$

where $\quad \lambda=4 c\left(c+\varepsilon \cos \phi_{1}\right), \quad \delta^{2}=(b-\varepsilon)^{2}-a^{2}+4 b \varepsilon \sin ^{2}(\phi$ $\left.-\phi_{1}\right) / 2$, and $c$ is dominant when compared to $b \cos \phi$. So we have the following integral for $n=7$ and 19 to evaluate

$$
I_{n}=\int_{0}^{2 \pi} \frac{d \phi}{\delta^{n}\left(\lambda+\delta^{2}\right)^{n / 2}} .
$$

We now substitute the values for $\lambda$ and $\delta$ into this integral and we let $x=\phi / 2$. Note that we have set $\phi_{1}=0$ since as previously shown gravity is negligible, so that in response to the centrifugal and the Lennard-Jones energies alone the orbiting body's position will be in the plane of rotation, and we have 


$$
I_{n}=2 \int_{0}^{\pi} \frac{d x}{\left(\mu+\nu \sin ^{2} x\right)^{n / 2}\left(\sigma+\nu \sin ^{2} x\right)^{n / 2}}
$$

where $\nu=4 b \varepsilon, \sigma=\lambda+\mu, \mu=(b-\varepsilon)^{2}-a^{2}$, and $\lambda=4 c(c+\varepsilon)$. Splitting the integral again, letting $n=2 m$ and making the substitution $t=\cot x$ we obtain

$$
\begin{aligned}
I_{2 m} & =4 \int_{0}^{\pi / 2} \frac{\csc ^{4 m} x d x}{\left(\mu \csc ^{2} x+\nu\right)^{m}\left(\sigma \csc ^{2}+\nu\right)^{m}} \\
& =4 \int_{0}^{\infty} \frac{\left(t^{2}+1\right)^{2 m-1} d t}{\left(\mu t^{2}+\mu+\nu\right)^{m}\left(\sigma t^{2}+\sigma+\nu\right)^{m}} \\
& =\frac{4}{(\mu+\nu)^{m}(\sigma+\nu)^{m}} \int_{0}^{\infty} \frac{\left(t^{2}+1\right)^{2 m-1} d t}{\left(t^{2}+1-\alpha t^{2}\right)^{m}\left(t^{2}+1-\beta t^{2}\right)^{m}},
\end{aligned}
$$

where $\alpha=\nu /(\nu+\mu), \beta=\nu /(\nu+\sigma)$, and we are interested in the two values $m=7 / 2$ and $m=19 / 2$. Now on writing this integral in the form

$$
\begin{aligned}
I_{2 m}= & \frac{4}{(\mu+\nu)^{m}(\sigma+\nu)^{m}} \\
& \times \int_{0}^{\infty} \frac{\left(t^{2}+1\right)^{-1} d t}{\left[1-\alpha t^{2} /\left(t^{2}+1\right)\right]^{m}\left[1-\beta t^{2} /\left(t^{2}+1\right)\right]^{m}},
\end{aligned}
$$

we are again led to make the substitution Eq. (A4)

$$
\begin{aligned}
I_{2 m} & =\frac{4}{(\mu+\nu)^{m}(\sigma+\nu)^{m}} \int_{0}^{1} \frac{\left(1-z^{2}\right)^{-1 / 2} d z}{\left(1-\alpha z^{2}\right)^{m}\left(1-\beta z^{2}\right)^{m}} \\
& =\frac{2}{(\mu+\nu)^{m}(\sigma+\nu)^{m}} \int_{0}^{1} \frac{u^{-1 / 2}(1-u)^{-1 / 2} d u}{(1-\alpha u)^{m}(1-\beta u)^{m}},
\end{aligned}
$$

where the final line follows on making the substitution $u$ $=z^{2}$. From Bailey ${ }^{24}$ we have

$$
\begin{aligned}
& \int_{0}^{1} \frac{u^{\alpha-1}(1-u)^{\gamma-\alpha-1}}{(1-u x)^{\beta}(1-u y)^{\beta^{\prime}}} d u \\
& \quad=\frac{\Gamma(\alpha) \Gamma(\gamma-\alpha)}{\Gamma(\gamma)} F_{1}\left(\alpha ; \beta, \beta^{\prime} ; \gamma ; x, y\right),
\end{aligned}
$$

where $F_{1}$ is the first Appell hypergeometric function of two variables defined from Bailey ${ }^{24}$ by the double integral

$$
F_{1}\left(\alpha ; \beta, \beta^{\prime} ; \gamma ; x, y\right)=\sum_{m=0}^{\infty} \sum_{n=0}^{\infty} \frac{(\alpha)_{m+n}(\beta)_{m}\left(\beta^{\prime}\right)_{n} x^{m} y^{n}}{(\gamma)_{m+n} m ! n !},
$$

which is always convergent for $|x|<1,|y|<1$. The integral $I_{2 m}$ becomes

$$
I_{2 m}=\frac{2 \pi}{(\mu+\nu)^{m}(\sigma+\nu)^{m}} F_{1}\left(\frac{1}{2} ; m, m ; 1 ; \alpha, \beta\right),
$$

where $\alpha=\nu /(\mu+\nu), \beta=\nu /(\sigma+\nu)$ and here we are interested in the two values $m=7 / 2$ and $m=19 / 2$. The convergence criteria state that $|\alpha|<1$ and $|\beta|<1$, which reduce to the previously stated geometric criteria that $\varepsilon \leq b-a$. We note that in our case $\beta=\beta^{\prime}$ so that from Burchnall and Chaundy ${ }^{25}$ the following formula applies:

$$
\begin{aligned}
F_{1}(\alpha ; \beta, \beta ; \gamma ; x, y)= & \sum_{k=0}^{\infty} \frac{(\alpha)_{k}(\beta)_{k}(\gamma-\alpha)_{k} x^{k} y^{k}}{(\gamma)_{2 k} k !} F(\alpha+k, \beta \\
& +k ; \gamma+2 k ; x+y-x y) \\
= & \sum_{k=0}^{\infty} \frac{(-1)^{k}(\alpha)_{2 k}(\beta)_{k} x^{k} y^{k}}{(\gamma)_{2 k} k !} F(\alpha+2 k, \beta \\
& +k ; \gamma+2 k ; x+y) .
\end{aligned}
$$

In addition, from Gradshteyn and Ryzhik ${ }^{22}$ we can make the transformation

$$
\begin{aligned}
F_{1}(\alpha ; \beta, \beta ; \gamma ; x, y)= & (1-y)^{-\alpha} F_{1} \\
& \times\left(\alpha ; \beta, \gamma-2 \beta ; \gamma ; \frac{y-x}{y-1}, \frac{y}{y-1}\right),
\end{aligned}
$$

which results in one of the infinite summations in Eq. (B3) becoming finite. The resulting expression for the energy becomes

$$
\begin{aligned}
V_{1}= & 2 \pi^{2} a b c \eta_{b} \eta_{t}\left[-\frac{5 \pi A a^{3} \lambda^{3}}{(\mu+\nu)^{7 / 2}(\sigma+\nu)^{7 / 2}}\right. \\
& \times F_{1}\left(\frac{1}{2} ; \frac{7}{2}, \frac{7}{2} ; 1 ; \alpha, \beta\right)+\frac{2431 \pi B a^{9} \lambda^{9}}{32(\mu+\nu)^{19 / 2}(\sigma+\nu)^{19 / 2}} \\
& \left.\times F_{1}\left(\frac{1}{2} ; \frac{19}{2}, \frac{19}{2} ; 1 ; \alpha, \beta\right)\right],
\end{aligned}
$$

where $\nu=4 b \varepsilon, \quad \sigma=\lambda+\mu, \lambda=4 c(c+\varepsilon), \quad \mu=(b-\varepsilon)^{2}-a^{2}, \alpha$ $=\nu /(\mu+\nu)$, and $\beta=\nu /(\sigma+\nu)$. Or more simply

$$
\begin{aligned}
V_{1}= & 256 \pi^{3} a b c \eta_{b} \eta_{t}\left[-\frac{5 A a^{3} c^{3}(c+\varepsilon)^{3}}{2[\delta(4 c(c+\varepsilon)+\delta)]^{7 / 2}}\right. \\
& \times F_{1}\left(\frac{1}{2} ; \frac{7}{2}, \frac{7}{2} ; 1 ; \alpha, \beta\right)+\frac{155584 B a^{9} c^{9}(c+\varepsilon)^{9}}{[\delta(4 c(c+\varepsilon)+\delta)]^{19 / 2}} \\
& \left.\times F_{1}\left(\frac{1}{2} ; \frac{19}{2} \frac{19}{2} ; 1 ; \alpha, \beta\right)\right],
\end{aligned}
$$

where $\delta=(b+\varepsilon)^{2}-a^{2}, \alpha=4 b \varepsilon / \delta$, and $\beta=4 b \varepsilon /[4 c(c+\varepsilon)+\delta]$. We note that from kinematic or geometric constraints that this energy is only sensible for $|\varepsilon| \leq|b-a|$.

${ }^{1}$ S. Iijima, Nature 354, 56 (1991).

${ }^{2}$ M.-F. Yu, O. Lourie, M. J. Dyer, K. Moloni, T. F. Kelly, and R. S. Ruoff, Science 287, 637 (2000).

${ }^{3}$ J. Cumings and A. Zettl, Science 289, 602 (2000).

${ }^{4}$ Q. Zheng and Q. Jiang, Phys. Rev. Lett. 88, 1 (2002).

${ }^{5}$ S. B. Legoas, V. R. Coluci, S. F. Braga, P. Z. Coura, S. O. Dantas, and D. S. Galvao, Phys. Rev. Lett. 90, 055504 (2003).

${ }^{6}$ L. A. Girifalco, M. Hodak, and R. S. Lee, Phys. Rev. B 62, 13104 (2000).

${ }^{7}$ P. Liu, Y. W. Zhang, and C. Lu, J. Appl. Phys. 97, 1 (2005).

${ }^{8}$ M. Hodak and L. A. Girifalco, Chem. Phys. Lett. 350, 405 (2001).

${ }^{9}$ D. Qian, W. K. Liu, and R. S. Ruoff, J. Phys. Chem. B 105, 10753 (2001).

${ }^{10}$ B. J. Cox, N. Thamwattana, and J. M. Hill, Proc. R. Soc. London, Ser. A 463, 461 (2007).

${ }^{11}$ J. Liu, H. Dai, J. H. Hafner, D. T. Colbert, R. E. Smalley, S. J. Tans, and C. Dekker, Nature 385, 780 (1997).

${ }^{12}$ M. Sano, A. Kamino, J. Okamura, and S. Shinkai, Science 293, 1299 (2001).

${ }^{13}$ R. Martel, H. R. Shea, and P. Avouris, Nature 398, 299 (1999). 
${ }^{14}$ R. Martel, H. R. Shea, and P. Avouris, J. Phys. Chem. B 103, 7551 (1999).

${ }^{15}$ M. Huhtala, A. Kuronen, and K. Kaski, Comput. Phys. Commun. 147, 91 (2002).

${ }^{16}$ J. Han, Toroidal Single Wall Carbon Nanotubes in Fullerene Cop Circles, NAS Technical Report (1997).

${ }^{17}$ M. S. Dresselhaus, G. Dresselhaus, and R. Saito, Carbon 33, 883 (1995)

${ }^{18}$ Y. Wang, D. Tománek, and G. F. Bertsch, Phys. Rev. B 44, 6562 (1991).

${ }^{19}$ L. A. Girifalco, J. Phys. Chem. 96, 858 (1992).

${ }^{20}$ B. J. Cox, N. Thamwattana, and J. M. Hill, Proc. R. Soc. London, Ser. A 463, 477 (2007).
${ }^{21}$ F. D. Colavecchia, G. Gasaneo, and J. E. Miraglia, Comput. Phys. Commun. 138, 29 (2001).

${ }^{22}$ I. S. Gradshteyn and I. M. Ryzhik, Table of Integrals Series and Products, 6th ed. (Academic, New York, 1965).

${ }^{23}$ A. Erdélyi, W. Magnus, F. Oberhettinger, and F. G. Tricomi, Higher Transcendental Functions (McGraw-Hill, New York, 1953), Vol. 1, Chap. 2, pp. 56-119.

${ }^{24}$ W. N. Bailey, Generalized Hypergeometric Series (Hafner, New York, 1972).

${ }^{25}$ J. L. Burchnall and T. W. Chaundy, Q. J. Math. 11, 249 (1940). 